\title{
A novel mutation in the SERAC1 gene correlates with the severe manifestation of the MEGDEL phenotype, as revealed by whole-exome sequencing
}

\author{
MERYEM ALAGOZ ${ }^{1}$, NASIM KHERAD ${ }^{1}$, SELDA TURKMEN ${ }^{2}$, \\ HATICE BULUT ${ }^{3}$ and ADNAN YUKSEL ${ }^{3}$ \\ ${ }^{1}$ Department of Molecular Biology and Genetics, Genome Centre, Biruni University, Istanbul 34010; \\ ${ }^{2}$ Department of Medical Biology, Istanbul Cerrahpasa University, Istanbul 34096; \\ ${ }^{3}$ Faculty of Medicine, Biruni University Hospital, Istanbul 34010, Turkey
}

Received May 23, 2019; Accepted January 10, 2020

DOI: $10.3892 /$ etm.2020.8658

\begin{abstract}
The condition 3-methylglutaconic aciduria (3-MGA) with deafness, encephalopathy and Leigh-like (MEGDEL) syndrome, also known as 3-MGA IV, is one of a group of five rare metabolic disorders characterized by mitochondrial dysfunction, resulting in a series of phenotypic abnormalities. It is a rare, recessive inherited disorder with a limited number of cases reported worldwide; hence, it is important to study each case to understand its genetic complexity. An impaired activity of serine active site-containing protein 1 (SERAC1), caused by mutations, leads to defects in phosphatidylglycerol remodelling, which is important for mitochondrial function and intracellular cholesterol trafficking. In the present study, the patients (two male siblings of consanguineous Turkish parents) were analysed, whose multisystem dysfunctions, including an elevated 3-MGA concentration in early age, hearing loss and Leigh-like syndrome as determined by MRI, were consistent with MEGDEL syndrome. A novel mutation in the SERAC1 gene, in the upstream lipase domain, c.1015G>C (p.Gly339Arg) mutation located on exon 10 of the SERAC1, was identified and predicted to cause protein dysfunction. Furthermore, the results pointed towards a possible association between this mutation and the severity of MEGDEL syndrome.
\end{abstract}

\section{Introduction}

The condition 3-methylglutaconic aciduria (3-MGA) with deafness, encephalopathy and Leigh-like (MEGDEL)

Correspondence to: Professor Meryem Alagoz, Department of Molecular Biology and Genetics, Genome Centre, Biruni University, 10 Y1l Street, Istanbul 34010, Turkey

E-mail:malagoz@biruni.edu.tr

Key words: 3-methylglutaconic aciduria with deafness, encephalopathy and Leigh-like syndrome, serine active site-containing protein 1, SERAC1 gene, whole-exome sequencing, mutation, rare disease syndrome, also known as type IV 3-MGA [Mendelian of Inheritance in Man (MIM) \#614739], is associated with a heterogeneous group of disorders, including MGA, deafness, encephalopathy and Leigh-like lesions (1) as observed on MRI scans, progressive spasticity, dystonia (2-6), brain atrophy and neonatal liver disease (4), followed by increasing levels of enzymatic activity in the liver (7). Type IV 3-MGA, in contrast to the other types (I, II, III and V), is based on undefined clinical subtypes and the underlying aetiology has yet to be elucidated, even though affected individuals have been indicated to have heterogeneous genotypes, similar to those in the other groups $(8,9)$. Since elevated levels of liver enzymes have been observed to be a phenotype characteristic of MEGDEL syndrome, the term 'MEGDEL' was suggested in order to include all of the phenotypic disorders that occur most frequently in affected patients (4). MEGDEL syndrome is a rare, recessive inherited disorder, the fundamental underlying causes of which are genetic impairments attributable to multiple complexities, including DNA demolition syndromes and mitochondrial DNA deletion syndromes, as well as mitochondrial myopathy, encephalopathy, lactic acidosis and stroke syndrome. Dysregulation of 3-MGA in all five types has been identified on the basis of genetic pathogenic variants. Type I 3-MGA (MIM \#250950) has been reported to result from a mutation in the AU RNA binding methylglutaconyl-CoA hydratase gene, which causes a deficiency in 3-methylglutaconyl-CoA hydratase, the enzyme involved in L-leucine degradation (7). Type II 3-MGA, also known as Barth syndrome (10), is characterized by oxidative phosphorylation dysfunction, neutropenia and cardiomyopathy, which lead to defective cardiolipin remodelling $(8,11)$ due to a mutation in the tafazzin (TAZ) gene on chromosome X $(12,13)$. The TAZ gene encodes the TAZ protein, the enzymatic function of which is to regulate cardiolipin remodelling in order to maintain its level in the inner mitochondrial membrane $(11,14,15)$. Type III 3-MGA, also known as Costeff syndrome, is a rare neurogenic autosomal recessive inherited disorder $(16,17)$ that is caused by mutations in the outer mitochondrial membrane lipid metabolism regulator OPA3 gene, whose function remains to be fully elucidated (18). Patients with this syndrome have 
been reported to exhibit optic atrophy, followed by motor deficits and ataxia (16). Type V 3-MGA, also known as dilated cardiomyopathy with ataxia syndrome (19), is an autosomal recessive disorder with a defect in the DnaJ heat shock protein family (Hsp40) member C19 gene that leads to deficiencies in muscle strength, anaemia and growth retardation $(19,20)$. MEGDEL syndrome is caused by biallelic pathogenic variants in the Serine active site-containing 1 (SERAC1) gene, which is located on chromosome 6q25.3. SERAC1 protein, with the phenotypic MIM number 614725, belongs to the PGAG-like protein family, containing 654 amino acids and comprising a serine lipase domain which is hypothesized to fulfil a crucial role in protein and mitochondrial function. Furthermore, SERAC1 has a role in intracellular cholesterol trafficking and anomalous functioning of the protein affects phospholipid mechanisms of action (3). The protein is assumed to be responsible for phospholipid exchange at the mitochondrial interface and endoplasmic reticulum, and defects in this process lead to the phenotypic abnormalities that are characteristic of MEGDEL syndrome.

A range of pathogenic variants that have been observed in patients with MEGDEL syndrome have been identified and confirmed by exome sequencing and Sanger sequencing $(4,5,21)$. Through these studies, the majority of the SERAC1 gene pathogenic variants have been categorized as frameshift, nonsense or missense pathogenic variants within or upstream of the lipase domain $(3,4,8)$.

The aim of the present study was to investigate the genes involved in the onset of MEGDEL syndrome and elucidate the association between novel variants and phenotypic outcome of the disease. Since the genetic complexity of MEGDEL syndrome has yet to be completely elucidated, detailed studies are required to identify further pathogenic variants that lead to the phenotypic characteristics of the disorder. In the present study, biochemical analysis and MRI scans were performed to confirm the primary diagnosis with MEGDEL syndrome. Whole exome sequencing (WES) analyses were performed to identify pathogenic variants associated with the syndrome and the mutations were analysed with respect to the patients' family to confirm the pattern of inheritance. The results obtained from the present study suggested that this mutation influences the severity of the disease, causing severe developmental delays. Compared to previous studies using brain MRI of patients with MEGDEL syndrome representing bilateral basal ganglia alterations and dystonia, the present study observed a greater impact of the mutation on the brain lobes. This resulted in cerebellar atrophy, corpus callosum thinning and lateral ventricle expansion as observed using MRI.

\section{Patients and methods}

Patients. Two male siblings, born in 2013 and 2015, from consanguineous Turkish parents were admitted to Biruni Hospital (Istanbul, Turkey) with psychomotor retardation, spasticity, dystonia and deafness.

Brain MRI scanning. MRI scans were performed for regular patient care following admission to the hospital. Different pulse sequences were used for the MRI procedure, and
T1- and T2-weighted images were available for the younger sibling.

WES by next-generation sequencing (NGS) and subsequent bioinformatics analysis. WES was performed to analyse the coding exons and the exon-intron boundaries of protein-coding genes. Genomic DNA preparation, exome capture and Illumina ${ }^{\circledR}$ sequencing (NextSeq500 platform) were performed according to the manufacturer's instructions. In brief, genomic DNA (gDNA) was extracted from the whole peripheral blood sample using an Invitrogen ${ }^{\circledR}$ NextSeq500 iPrep PureLink gDNA blood kit (Thermo Fisher Scientific, Inc.). The genomic library was prepared using an Agilent SureSelect Target Enrichment system (Agilent Technologies, Inc.). Enrichment of coding exons and flanking intronic regions was performed using the Agilent SureSelect Human All Exon V6 reagent (Agilent Technologies, Inc.), following the manufacturer's protocol and as previously described (22), and sequencing was performed using an Illumina ${ }^{\circledR}$ NextSeq500 system (Illumina, Inc.). Sequences were mapped to the human genome (GRCh37/hg19) using the Burrows-Wheeler Aligner (version 0.6.1; algorithm 'BWA-SW'; default parameters) to obtain targeted sequencing data. Variants with a frequency $>1 \%$ in the population were removed from the collected data. Variants were subsequently annotated using Alamut ${ }^{\circledR}$ Visual (a decision-support software dedicated to variant diagnostics used by clinical and research molecular laboratories worldwide; see https://www. interactive-biosoftware.com/alamut-visual/), and the allele frequency was determined using the following databases: The National Center for Biotechnology Information database for nucleotide variations dbSNP (https://www.ncbi.nlm. nih.gov/snp), the exome aggregation consortium (ExAC) and the 1000 Genomes Project (https://www.internationalgenome.org/). Disease causality was assessed using ClinVar (https://www.ncbi.nlm.nih.gov/clinvar/) and exome sequencing project (ESP) variants, ExAC variants and ESP variants.

Sanger sequencing of the identified mutation. DNA from the patients and the parents was isolated from whole blood using the QIAamp DNA Blood Mini kit (Qiagen, Inc.). Exon 10 of the SERAC1 gene were amplified using primer sets (forwards, 5'-TCCAACCAAGAGCTAAGCAG-3'; and reverse, 5'-TGAA CATATCATGAGGGGTAGAG-3') and MyTaq $^{\mathrm{TM}}$ DNA Polymerases Mix (Bioline Reagents Ltd.). PCR was performed with $40 \mathrm{ng}$ of genomic DNA in $30 \mu \mathrm{l}$, with an initial denaturation at $94^{\circ} \mathrm{C}$ for $3 \mathrm{~min}, 30$ cycles of $94^{\circ} \mathrm{C}$ for $30 \mathrm{sec}, 55^{\circ} \mathrm{C}$ for $45 \mathrm{sec}$ and $72^{\circ} \mathrm{C}$ for $2 \mathrm{~min}$, followed by a final extension at $72^{\circ} \mathrm{C}$ for $10 \mathrm{~min}$. The SERAC1 gene was sequenced directly from purified PCR products using a BigDye Terminator Cycle Sequencing kit (version 3.1; Applied Biosystems; Thermo Fisher Scientific, Inc.) prior to analysis on an ABI 3130 automated DNA sequencer (Applied Biosystems; Thermo Fisher Scientific, Inc.).

Determination of biochemical parameters. Standard biochemical and metabolic tests (see the Results section for further details) were performed for the patients during the first month of life (note that the second of the siblings was admitted to the 
intensive care unit of the Biruni Hospital 22 days following birth), and then subsequently at the ages of 16 months and 3 years.

Computational analysis of protein structure and severity of damage caused by mutation. Protein structure analysis was performed using SWISS-MODEL exPASy (https://swissmodel. expasy.org/interactive) to determine the effect of the mutation on the SERAC1 protein. In addition, damage sensitivity of the mutation was analysed using PolyPhen2 (http://genetics.bwh. harvard.edu/pph2/) to investigate its role in the severity of the phenotypic characteristics. The mutation taster program (www.mutationtaster.org/) was used to predict the effects of the mutation on the protein.

\section{Results}

Patients data and biochemical analysis. The proband, a male, was the second child of a 22-year-old mother who married a first-degree relative (cousin). He was born in June 2013 with a neonatal weight of 2,900 g. The levels of neonatal thyroid-stimulating hormone (TSH) and biotinidase were normal and the phenylketonuria screening test was also negative on the 5th day of life. When the infant was 22 days old; however, the patient was admitted to an intensive care unit for 22 days due to asphyxia after birth. The blood test performed on 20th day of age revealed elevated levels of aspartate aminotransferase (AST), bilirubin, chlorine, magnesium, sodium, potassium, creatine kinase, lactate dehydrogenase (LDH) and TSH. At the time of the blood test, the urea concentration was also within the normal range. At the age of 16 months, the patient's biochemical analysis indicated an elevated level of 3-MGA, which compelled physicians to perform further investigations, including MRI scan. The patient was indicated to have hearing loss at the age of 21 months and strabismus was detected in the right eye. Furthermore, the clinical biochemistry screen revealed that the levels of AST, alanine aminotransferase, alkaline phosphatase and creatinine were above the normal range. The biochemical test also revealed that the lactate level was above the reference range. The TSH level, however, was within the normal range during the same period. Essential minerals, including sodium, potassium, calcium, magnesium, phosphorus and cholesterol, were revealed to have higher values compared with the reference range. At the same time, the biochemistry test results suggested that the uric acid, total protein, albumin and urea levels were below the normal range. The lactic acid level was close to the normal range (4.5-19.8), a result that differed from the previous test, and the level of vitamin B12 was above the reference range. The ferritin level was also below the reference range. The results of the biochemical analysis performed subsequently, when the patient was 3 years old, revealed that glucose and lactate levels were above their normal limits. An investigation was performed using tandem mass spectrometry, which determined that the arginine level was above the reference range. Biochemistry analyses also revealed that the creatine level was low, whereas the levels of AST and LDH were higher compared with the reference values. The analysis of the patient's organic acids revealed that the levels of 3-hydroxypropionic acid, pyruvic acid, 3-hydroxybutyric acid, ethyl malonic acid, adipic acid, 2-hydroxyglutaric acid, methylglutaric acid and 3-methylglutaconic acid in the patient's urine sample were higher than the reference levels (Table SI).

Brain MRI for diagnosis of encephalopathy and neurological analysis. Electroencephalography was performed on the patient to determine the level of epileptic activity in the occipital region. Muscle biopsy revealed the presence of myopathic fibres in muscle tissue. The results of the echocardiogram were normal. However, brain MRI scans of the patient revealed abnormalities in different sections of the brain. Axial T2-weighted MRI revealed an expansion of the cerebellar follicles and 4th ventricle, located in the upper part of the medulla, causing cerebellar atrophy (Fig. 1A and B). In addition to the above observation, increased intensities of the bilateral caudate nucleus (Fig. 1C) and bilateral symmetric signal (Fig. 1D) were identified and expansion of lateral ventricles had occurred (Fig. 1E), causing putamen volume loss. Hypoplasia of the inferior vermis, observed in the sagittal T2-weighted MRI scan, resulted in thinning and atrophy of the corpus callosum (brain stem; Fig. 1F). Fluid-attenuated inversion recovery analysis of the bilateral basal ganglia and temporal lobe revealed a signal intensity that may be attributed to the involvement of white matter (Fig. $1 \mathrm{G}$ and $\mathrm{H}$ ).

\section{Genetic analysis}

Determination of pathogenic variants in the patient using $N G S$. WES revealed 13,520 genomic variants, which were filtered from $>100$ reads. Analysis of the distribution of types and frequencies of sequence variants revealed that $50.17 \%$ were intron variants, $12.95 \%$ were missense variants, $13.35 \%$ were synonymous variants, $18.43 \%$ were downstream gene variants, $8.29 \%$ were non-coding exon variants, $2.35 \%$ were upstream gene variants, $1.16 \%$ were splice region variants, $0.27 \%$ had in-frame insertions and $0.21 \%$ were missense variant splice region variants (Fig. 2A). Amongst the variants, certain pathogenic variants were identified, including NOC2-like nucleolar associated transcriptional repressor [NOC2L; single nucleotide variation (SNV), NM_015658.3:c.1443+455_1443+457delCTG], DFFB (NM_004402.2:c.782+58_782+59 insCGGCCC), cyclin D kinase (CDK) 11B (NM_033487.1:c.-167+273T>C), nephrocystin 4 (NPHP4; NM_015102.3:c.3131G>A, NP_055917.1:p. Arg1044His) and SERAC1. WES detected 13 different pathogenic variants in the SERAC1 gene, 11 of which were indicated to be associated with the disease (Table I). Analysis of the 2 variants that were detected in the SERAC1 gene using Alamut ${ }^{\circledR}$ Visual software suggested that mutation-associated pathogenicity linked to the disorder was present in a homozygous mutation at c.1015G>C (p.Gly339Arg) (Fig. 2B).

Identification of novel pathogenic variants in SERAC1. As the WES results revealed a novel SNV c.1015G>C (p.Gly339Arg) mutation located on exon 10 of the SERAC1 gene in the proband, confirmation analysis was performed on the patient to determine the presence of the mutation. Patient genotype confirmation and familial co-segregation analysis were performed. All coding exons and exon-intron boundaries of the SERAC1 gene were amplified using primer sets designed by a previous study (9). In order to confirm the presence of the 
Table I. Variants detected in the serine active site-containing 1 gene using whole-exome sequencing.

\begin{tabular}{|c|c|c|c|c|c|}
\hline $\begin{array}{l}\text { Mutation } \\
\text { type }\end{array}$ & $\begin{array}{l}\text { Reference sequence } \\
\text { mRNA(NM) }\end{array}$ & $\begin{array}{l}\text { Mutation } \\
\text { location }\end{array}$ & $\begin{array}{l}\text { Mutation } \\
\text { type }\end{array}$ & $\begin{array}{l}\text { Reference } \\
\text { SNP (rs) }\end{array}$ & $\begin{array}{l}\text { Variant } \\
\text { type }\end{array}$ \\
\hline SNV & NM_032861.3 & c. $1403+537 \mathrm{C}>\mathrm{G}$ & Homozygous & rs977794 & Intron variant \\
\hline SNV & NM_032861.3 & c. $1016-2838 \mathrm{~A}>\mathrm{G}$ & Homozygous & rs 12523959 & Intron variant \\
\hline $\mathrm{SNV}^{\mathrm{a}}$ & NM_032861.3 & c. $1015 \mathrm{G}>$ Cp.Gly339Arg & Homozygous & $?$ & $\begin{array}{l}\text { Missense variant, } \\
\text { splice region variant }\end{array}$ \\
\hline SNV & NM_032861.3 & c. $738+6201 \mathrm{C}>\mathrm{T}$ & Homozygous & rs 12197370 & Intron variant \\
\hline SNV & NM_032861.3 & c. $738+4454 \mathrm{~A}>\mathrm{C}$ & Homozygous & rs844140 & Intron variant \\
\hline SNV & NM_032861.3 & c. $356-216 \mathrm{C}>\mathrm{T}$ & Homozygous & rs9365928 & Intron variant \\
\hline SNV & NM_032861.3 & c. $355+300 \mathrm{~A}>\mathrm{G}$ & Homozygous & rs9356398 & Intron variant \\
\hline SNV & NM_032861.3 & c. $355+297 \mathrm{C}>\mathrm{T}$ & Homozygous & rs9364823 & Intron variant \\
\hline SNV & NM_032861.3 & c. $355+11 \mathrm{~A}>\mathrm{G}$ & Homozygous & rs9356399 & Intron variant \\
\hline SNV & NM_032861.3 & c. $249 \mathrm{C}>\mathrm{T}$ & Homozygous & rs6929274 & Synonymous variant \\
\hline SNV & NM_032861.3 & c. $129-34 C>G$ & Homozygous & rs6929520 & Intron variant \\
\hline SNV & NM_032861.3 & c. $92-334 \mathrm{G}>\mathrm{T}$ & Heterozygous & $?$ & Intron variant \\
\hline SNV & NM_032861.3 & c. $-1-4619 \mathrm{G}>\mathrm{A}$ & Homozygous & rs76958658 & Intron variant \\
\hline
\end{tabular}

${ }^{a}$ The mutation was detected in the patient was not previously reported. SNV, single nucleotide variant. SNPs, single nucleotide polymorphisms. ?, rs number is not given.
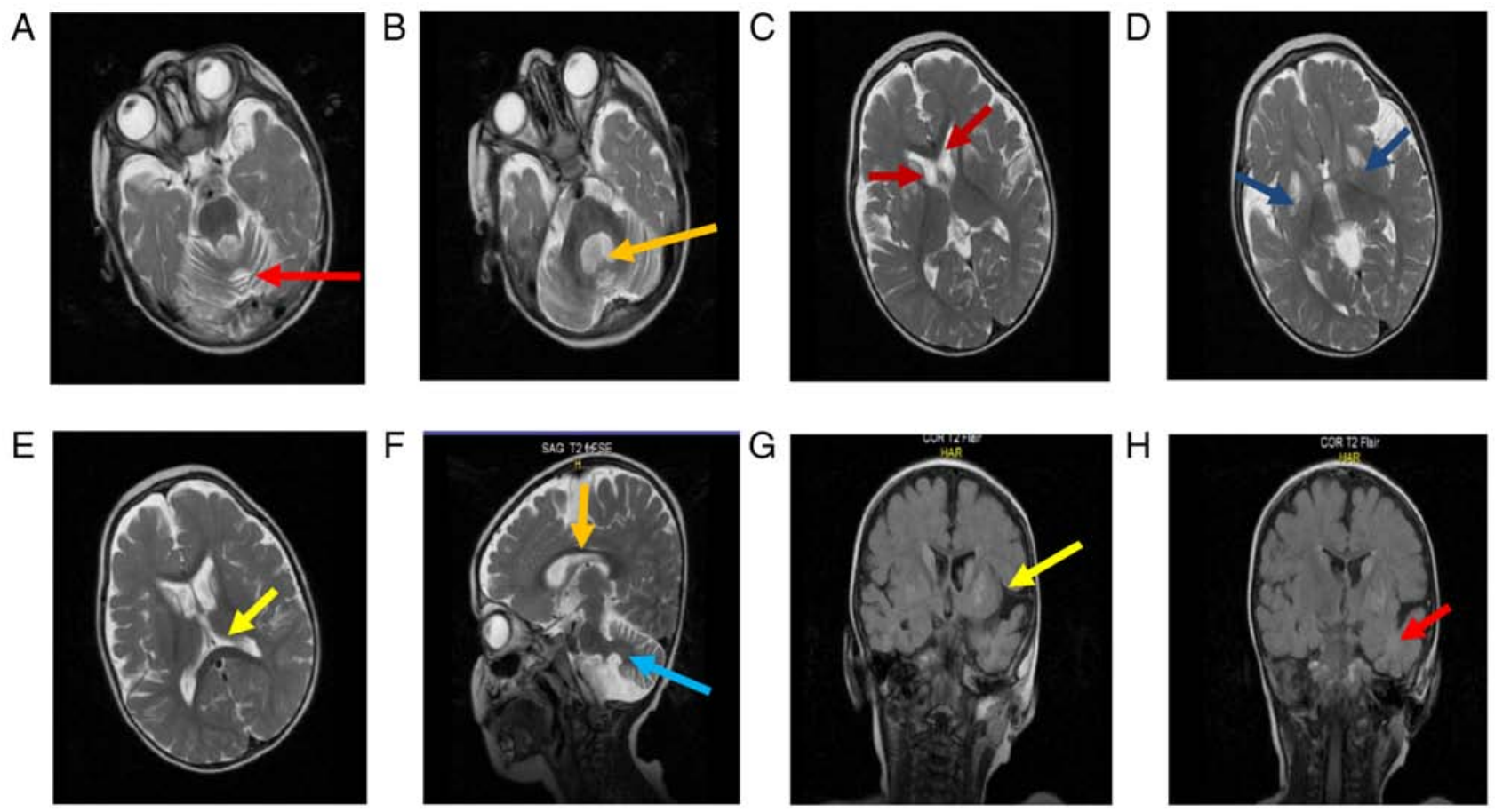

Figure 1. T2-weighted MRI examinations of the patient. An axial brain examination revealed (A) cerebellar atrophy due to expansion of the cerebellar follicles (denoted by the red arrow) and (B) an abnormally broadened 4th ventricle (indicated by the yellow arrow). Putamen examination, revealing (C) a loss of putamen volume due to increasing intensity of bilateral the caudate nucleus (red arrows) and (D) bilateral symmetric signal (blue arrows), followed by (E) expanded secondary ventricles (indicated by the yellow arrow). (F) The sagittal examination revealed thinning of the corpus callosum due to corpus callosum atrophy (orange arrow) and an abnormal view of the 4th ventricle due to hypoplasia of inferior vermis (blue arrow). A coronal FLAIR examination was used to detect signal intensity in $(\mathrm{G})$ the bilateral basal ganglia (indicated by the yellow arrow) and, particularly, $(\mathrm{H})$ in the temporal lobes, where the red arrow denotes the involvement of white matter. FLAIR, fluid-attenuated inversion recovery; SAG, sagittal; COR, coronal.

novel c.1015G>C (p.Gly339Arg) mutation located on exon 10 of the SERAC1 gene and its inheritance pattern, co-segregation analysis was performed on the proband, the proband's sibling and the parents by Sanger sequencing. The results confirmed the pathogenic variants to be homozygous in the proband and the proband's affected brother, who inherited one mutant allele 
A

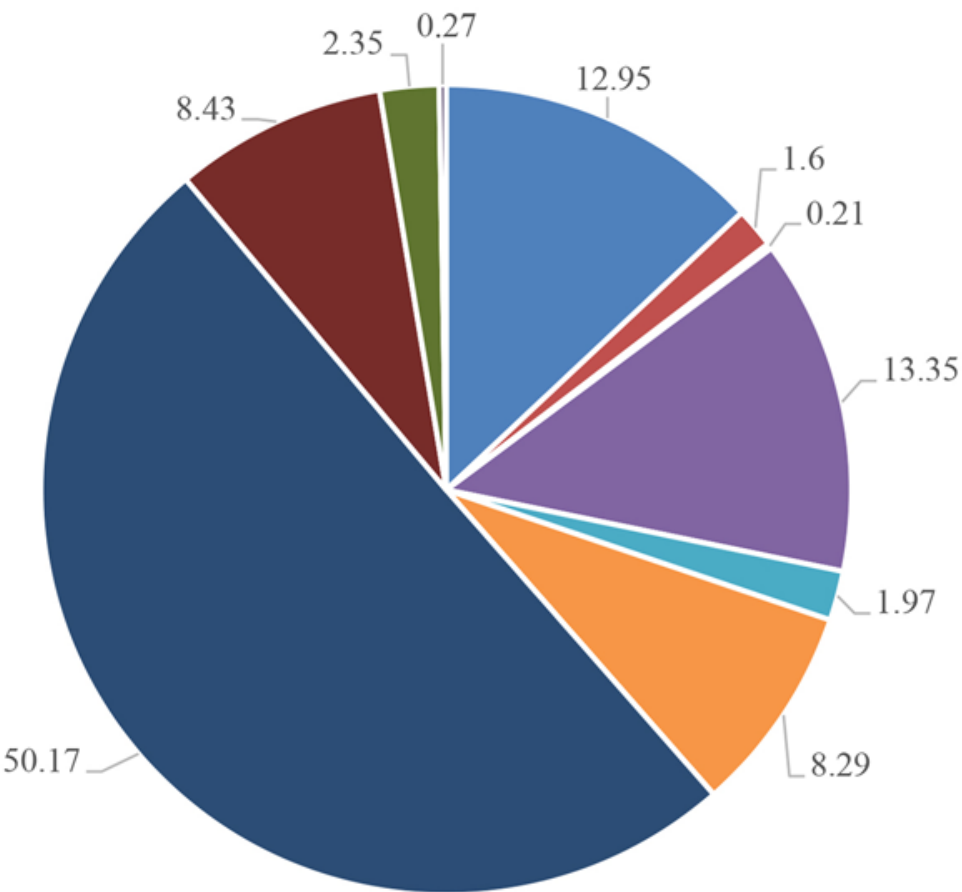

- Missense variant

- Splice region variant

- Missense variant, splice region variant

- Synonymous variant

- Downstream gene variant

- Noncoding exon variant

- Intron variant

- Downstream gene variant

- Upstream gene variant

- Inframe insersion

B SERAC1 - Serine active site containing 1 | GRCh38 (Chr 6)

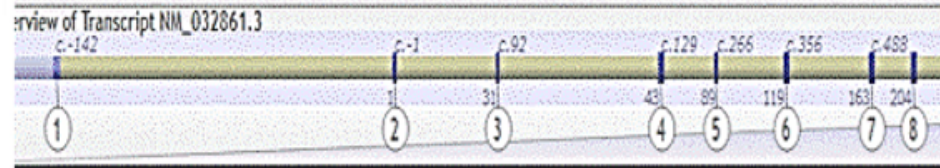

$\downarrow$ Genome - chr6:158,128,210-158,128,005 (GRCh38) - 206 bps

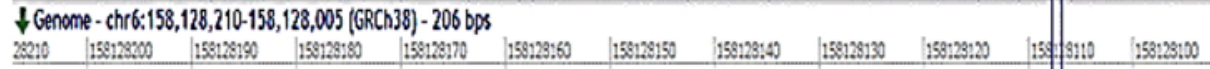
GCTGAAGTGTICCTGACGGGATTICATGICTCTITRTAITACGCACAGTAACCTITATACCGAAACTTACTTGTAGAAGTAAGAAGATATCAAGCGAGT CATTGTCGAAATATRIA CGACTTCACAAGGACTGCCCTAAAGTACAGAGAAATATAATGCGTGTCATTGGAAATATGGCTTTGAATGAACATCTTCATTCTTCTATAGTTCGCTCASDTAACAGCTTTATATS

I Hucleotide Conservation $\{0\}$<smiles>[GeH2]=[GeH2]</smiles>

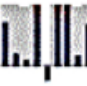

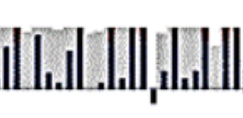

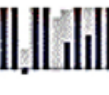
Hillill

I HM 032861.3: Homo supiens serine active site containing 1 (SERAC1), transcript variant 1, mRKA. (7) of

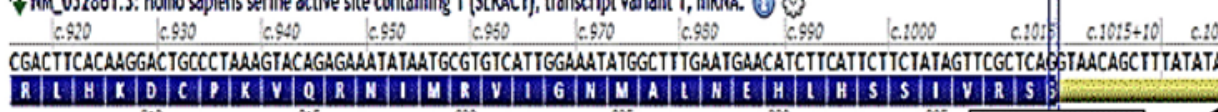

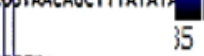

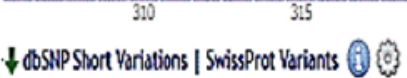
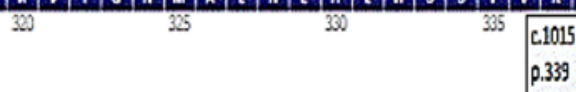

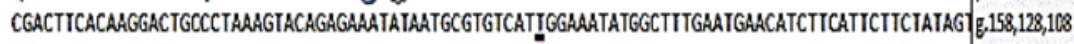

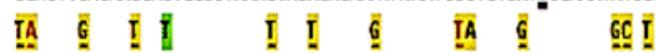

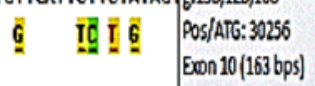

(Right dick for options)

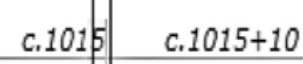

\begin{tabular}{l|l|l|l|l}
$\mathbf{V}$ & $\mathbf{R}$ & $\mathbf{S}$ & $\mathrm{B}$ \\
\hline
\end{tabular}

c. 1015

p. 339

AGTg.158,128,108

POS/ATG: 30256

Exon 10 (163 bps)

(Right click for options)

Figure 2. Mutation analysis by whole-exome sequencing. (A) In the present case, the types and frequencies of disease-causing mutations, and their distribution, are displayed in the pie chart. (B) The mutation pathogenicity of one of them was indicated to be biallelic at c.1015G>C (p.Gly339Arg), according to Alamut $^{\circledR}$ Visual software analysis.

from each heterozygous parent (Fig. 3A). Using sequencing data and information collected from family members, the hereditary pattern of MEGDEL syndrome in the family was illustrated in a pedigree chart that was performed on the patients' and the family's blood samples. The parents were both found to be a carrier while the children both manifested the disease and were affected (Fig. 3B). Analysis of the mutation location on the SERAC1 gene revealed the approximate position of the mutation to be upstream in a serine-lipase domain (Fig. 3C), which was downstream of the Armadillo-like helical domain. The mutation was predicted to be a missense variant. However, the data that were collected from the
Alamut analysis revealed the position of the mutation to be at the border of exon 10-intron 10 of the SERAC1 gene, and from this analysis, it was predicted to be a splice-site variant. The mutation, identified by PolyPhen2, was considered to be a causative factor of abnormality in intracellular cholesterol trafficking. The results of the bioinformatics protein structure analysis by SWISS-MODEL exPASy revealed changes in the conformation of the protein and in its binding sites, suggesting that the detected mutation was located in the upstream lipase domain, and may cause impaired protein-protein interaction (Fig. S1A and B). The analysis of the G339R substitution by damage sensitivity program suggested that the mutation is 
A

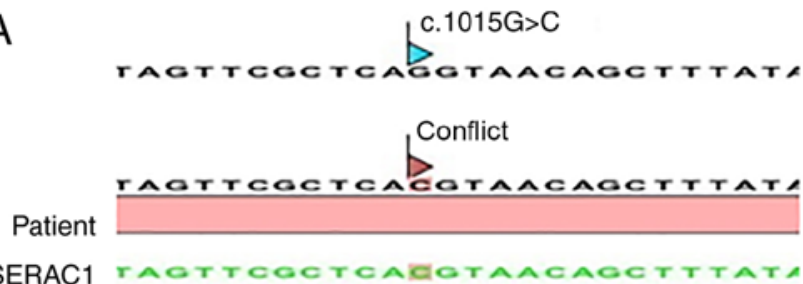

SERAC1 TAOTTCOCTCACOTAACAOCTTTATA

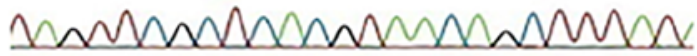
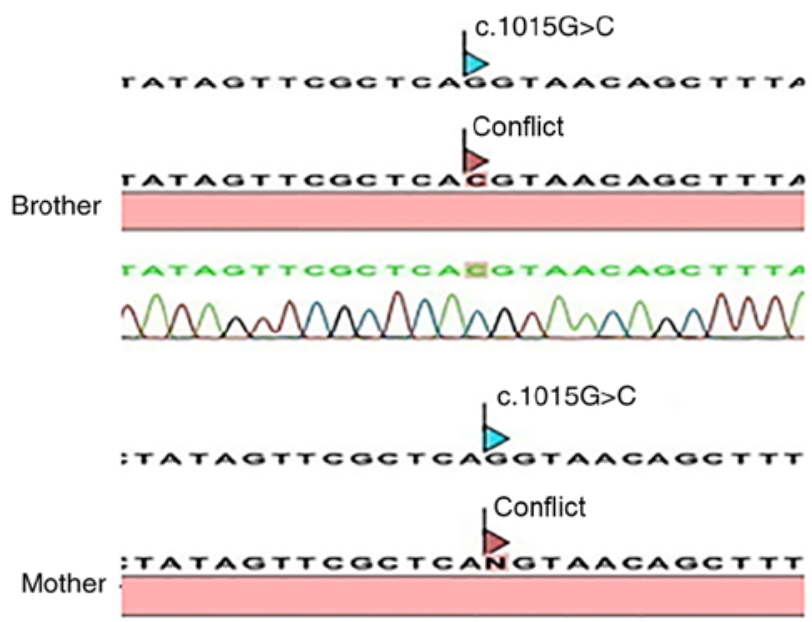

:TATAOTTCOCTCAMOTAACAOCTTT

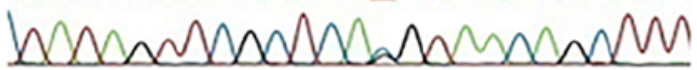

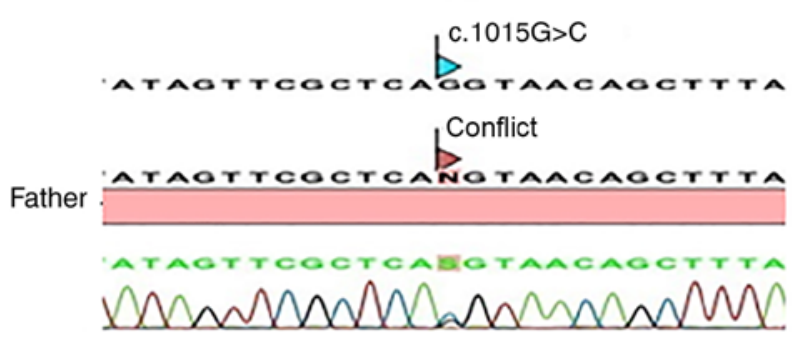

B

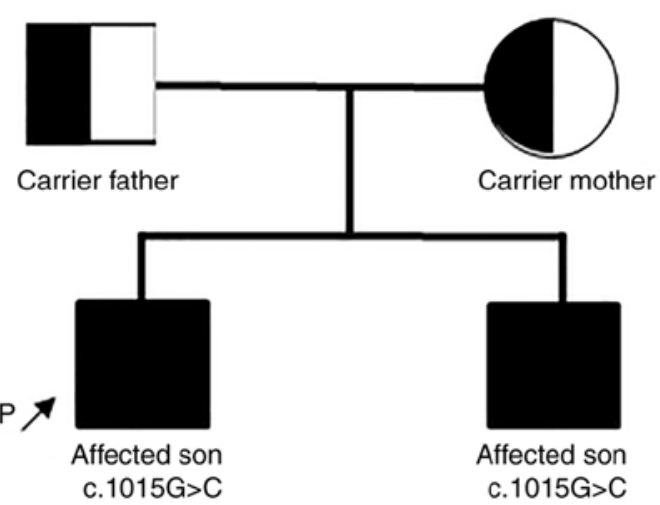

\section{Domains}

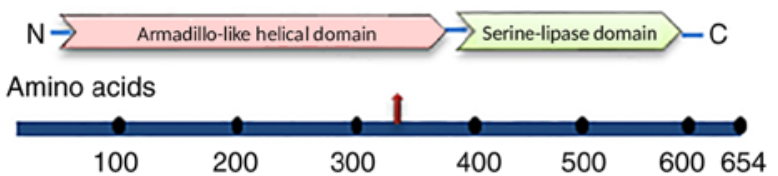

Figure 3. Confirmation of the novel SERAC1 mutation in the proband and the proband's family members, and its location in the SERAC1 protein domains. (A) Confirmation of the SERAC1 mutation by Sanger sequencing revealed that the proband and the proband's brother had homozygous mutations in the SERAC1 gene: Substitution of guanine at c.1015 by cytosine at p.1015 (c.1015G>C), resulting in an amino acid change from glycine to arginine (p.339). Co-segregation analysis of the parents by Sanger sequencing showed their heterozygosity for this novel mutation. The wild-type sequence of the SERAC1 gene is presented in the upper panels (black) and mutated sequences are in the bottom panels (green). (B) The pedigree chart was drawn to determine the inheritance pattern of the mutation in the proband's family. (C) The position of the c.1015G $>\mathrm{C}$ (p.Gly339Arg) mutation, which is located upstream of the serine-lipase domain, is illustrated (indicated by the red arrow). SERAC1, serine active site-containing 1.

likely to be damaging, with a score of 1.000. Furthermore, the mutation taster program suggested that the genetic impairment may cause alterations in the function of the SERAC1 protein and is disease-causing.

\section{Discussion}

Previous case studies identified pathogenic variants associated with the phenotypes of MEGDEL syndrome. In the present study, the characteristics of the disease were detected in both siblings, including the proband. The present study investigated the case of two siblings with 3-methylglutaconic aciduria and type IV 3-MGA with severe phenotypic characteristics of the disease in order to study the responsible genetic alteration in depth $(2,4,21)$. To date, 70 cases of MEGDEL syndrome have been reported worldwide. Since the clinical profiles of the affected siblings were entirely compatible with the characteristics of MEGDEL syndrome, the SERAC1 gene was selected as a candidate for mutation screening amongst genes, including NOC2L, DFFB, CDK11B and NPHP4, with certain pathogenic variants. The detection of a novel homozygous frameshift mutation in the SERAC1 gene confirmed the diagnosis of the syndrome and indicated the association of this gene with the phenotypic characteristics of MEGDEL syndrome.

Previous studies detected mutations in the Armadillo-like helical domain, serine-lipase domain and transmembrane domain of the SERAC1 protein, indicating the significance of each domain's function for protein stability $(1,3)$. The present study demonstrated that the location of the patient's mutation was upstream of the lipase domain and the localization of the mutation markedly increased damage sensitivity, causing SERAC1 protein dysfunction. Therefore, it may be inferred that this impairment has a crucial role in the onset of 
symptoms. Furthermore, the information obtained from the presence of a conserved lipase domain having the consensus lipase motif of GXSXG provides evidence highlighting the function of the serine lipase domain in lipid metabolism (3). Although MEGDEL syndrome is a phospholipid-remodelling disorder, it may also be classified as a disorder of intracellular cholesterol trafficking.

To summarize, the detailed investigation of the present study confirmed a novel mutation in the SERAC1 gene in the affected pedigree. This mutation is suggested to cause protein conformational changes that consequently result in protein dysfunction, which may lead to abnormalities in intracellular cholesterol trafficking. The present case has demonstrated several phenotypic characteristics of MEGDEL syndrome with a novel SERAC1 mutation. The 3-dimensional protein structure analysis revealed that the detected mutation may cause conformational changes in protein and binding sites, affecting protein-protein interaction. Furthermore, the other novel genetic alterations detected in the present case study may be responsible for the intense phenotypic characteristics of MEGDEL syndrome and require further investigation, which may lead to novel insight regarding the association of these pathogenic variants with the syndrome.

\section{Acknowledgements}

Not applicable.

\section{Funding}

No funding was received.

\section{Availability of data and materials}

The datasets used and/or analysed during the current study are available from the corresponding author on reasonable request.

\section{Authors' contributions}

MA performed the molecular genetic studies, and bioinformatics analysis of WES; participated in the design of the study; and drafted the manuscript. NK helped with the design of the study, performed data analysis and wrote the paper. ST helped with the data analysis. HB analysed MRI images. AY was involved in the collection of the patient's medical data and clinical diagnosis. All authors read and approved the final manuscript.

\section{Ethics approval and consent to participate}

Ethics approval was obtained from the Biruni University Ethics Committee (approval no. 2017/10-11). All procedures followed were in accordance with the ethical standards of the Helsinki Declaration of 1975, as revised in 2013.

\section{Patient consent for publication}

Informed consent was obtained from parents in order to publish the clinical data as well as images that were included in the study.

\section{Competing interests}

The authors declare they have no competing interests.

\section{References}

1. Roeben B, Schüle R, Ruf S, Bender B, Alhaddad B, Benkert T, Meitinger T, Reich S, Böhringer J, Langhans CD, et al: SERAC1 deficiency causes complicated HSP: Evidence from a novel splice mutation in a large family. J Med Genet 55: 39-47, 2018.

2. Wortmann S, Rodenburg RJ, Huizing M, Loupatty FJ, de Koning T, Kluijtmans LA, Engelke U, Wevers R, Smeitink JA and Morava E: Association of 3-methylglutaconic aciduria with sensori-neural deafness, encephalopathy, and Leigh-like syndrome (MEGDEL association) in four patients with a disorder of the oxidative phosphorylation. Mol Genet Metab 88: 47-52, 2006.

3. Wortmann SB, Vaz FM, Gardeitchik T, Vissers LE, Renkema GH, Schuurs-Hoeijmakers JH, Kulik W, Lammens M, Christin C, Kluijtmans LA, et al: Mutations in the phospholipid remodeling gene SERAC1 impair mitochondrial function and intracellular cholesterol trafficking and cause dystonia and deafness. Nat Genet 44: 797-802, 2012.

4. Sarig O, Goldsher D, Nousbeck J, Fuchs-Telem D, Cohen-Katsenelson K, Iancu TC, Manov I, Saada A, Sprecher E and Mandel $\mathrm{H}$ : Infantile mitochondrial hepatopathy is a cardinal feature of MEGDEL syndrome (3-methylglutaconic aciduria type IV with sensorineural deafness, encephalopathy and Leigh-like syndrome) caused by novel mutations in SER AC1. Am J Med Genet A 161 A: 2204-2215, 2013.

5. Maas RR, Iwanicka-Pronicka K, Kalkan Ucar S, Alhaddad B, AlSayed M, Al-Owain MA, Al-Zaidan HI, Balasubramaniam S, Barić I, Bubshait DK, et al: Progressive deafness-dystonia due to SERAC1 mutations: A study of 67 cases. Ann Neurol 82: 1004-1015, 2017.

6. Radha Rama Devi A and Lingappa L: Novel mutations in SERAC1 gene in two Indian patients presenting with dystonia and intellectual disability. Eur J Med Genet 61: 100-103, 2018.

7. IJlst L1: Loupatty FJ, Ruiter JP, Duran M, Lehnert W, and Wanders RJ: 3-Methylglutaconic aciduria type I is caused by pathogenic variants in AUH. Am J Hum Genet 71: 1463-1466, 2002.

8. Wortmann SB, Kluijtmans LA, Engelke UF, Wevers RA and Morava E: The 3-methylglutaconic acidurias: What's new? J Inherit Metab Dis 35: 13-22, 2012.

9. Wortmann SB, Rodenburg RJT, Jonckheere A, de Vries MC, Huizing $M$, Heldt $K$, van den Heuvel LP, Wendel U, Kluijtmans LA, Engelke UF, et al: Biochemical and genetic analysis of 3-methylglutaconic aciduria type IV: A diagnostic strategy. Brain 132: 136-146, 2009.

10. Houtkooper RH, Turkenburg M, Poll-The BT, Karall D, Pérez-Cerdá C, Morrone A, Malvagia S, Wanders RJ, Kulik W and Vaz FM: The enigmatic role of tafazzin in cardiolipin metabolism. Biochim Biophys Acta 1788: 2003-2014, 2009.

11. Xu Y, Kelley RI, Blanck TJ and Schlame M: Remodeling of cardiolipin by phospholipid transacylation. J Biol Chem 278: 51380-51385, 2003.

12. Bione S, D'Adamo P, Maestrini E, Gedeon AK, Bolhuis PA and Toniolo D: A novel X-linked gene, G4.5. is responsible for Barth syndrome. Nat Genet 12: 385-389, 1996.

13. Whited K, Baile MG, Currier P and Claypool SM: Seven functional classes of Barth syndrome mutation. Hum Mol Genet 22: 483-492, 2013.

14. Schlame M, Kelley RI, Feigenbaum A, Towbin JA, Heerdt PM, Schieble T, Wanders RJ, DiMauro S and Blanck TJ: Phospholipid abnormalities in children with Barth syndrome. J Am Coll Cardiol 42: 1994-1999, 2003.

15. Vreken P, Valianpour F, Nijtmans LG, Grivell LA, Plecko B, Wanders RJ and Barth PG: Defective remodeling of cardiolipin and phosphatidylglycerol in Barth syndrome. Biochem Biophys Res Commun 279: 378-382, 2000.

16. Costeff H, Elpeleg O, Apter N, Divry P and Gadoth N: 3-Methylglutaconic aciduria in 'optic atrophy plus'. Ann Neurol 33: 103-104, 1993.

17. Elpeleg ON, Costeff H, Joseph A, Shental Y, Weitz R and Gibson KM: 3-Methylglutaconic aciduria in the Iraqi-Jewish 'optic atrophy plus' (Costeff) syndrome. Dev Med Child Neurol 36: 167-172, 1994. 
18. Nystuen A, Costeff H, Elpeleg ON, Apter N, Bonné-Tamir B, Mohrenweiser H, Haider N, Stone EM and Sheffield VC: Iraqi-Jewish kindreds with optic atrophy plus (3-methylglutaconic aciduria type 3) demonstrate linkage disequilibrium with the CTG repeat in the $3^{\prime}$ untranslated region of the myotonic dystrophy protein kinase gene. Hum Mol Genet 6: 563-569, 1997.

19. Davey KM, Parboosingh JS, McLeod DR, Chan A, Casey R, Ferreira P, Snyder FF, Bridge PJ and Bernier FP: Mutation of DNAJC19, a human homologue of yeast inner mitochondrial membrane co-chaperones, causes DCMA syndrome, a novel autosomal recessive Barth syndrome-like condition. J Med Genet 43: 385-393, 2006.

20. Ojala T, Polinati P, Manninen T, Hiippala A, Rajantie J, Karikoski R, Suomalainen A and Tyni T: New mutation of mitochondrial DNAJC19 causing dilated and noncompaction cardiomyopathy, anemia, ataxia, and male genital anomalies. Pediatr Res 72: 432-437, 2012.
21. Tort F, García-Silva MT, Ferrer-Cortès X, Navarro-Sastre A, Garcia-Villoria J, Coll MJ, Vidal E, Jiménez-Almazán J, Dopazo J, Briones $\mathrm{P}$, et al: Exome sequencing identifies a new mutation in SERAC1 in a patient with 3-methylglutaconic aciduria. Mol Genet Metab 110: 73-77, 2013.

22. Bonnefond A, Philippe J, Durand E, Dechaume A, Huyvaert M, Montagne L, Marre M, Balkau B, Fajardy I, Vambergue A, et al: Whole-exome sequencing and high throughput genotyping identified KCNJ11 as the thirteenth MODY gene. PLoS One 7: e37423, 2012.

(i) (9) This work is licensed under a Creative Commons EY No ND Attribution-NonCommercial-NoDerivatives 4.0 International (CC BY-NC-ND 4.0) License. 